POS $\quad$ PROCEEDINGS

\title{
H.E.S.S. Observations of the Large Magellanic Cloud
}

\author{
Nukri Komin* \\ School of Physics, University of the Witwatersrand, Johannesburg, South Africa. \\ E-mail: nukri.komin@wits.ac.za
}

\section{Chia-Chun Lu}

Max-Planck-Institut für Kernphysik, Heidelberg, Germany.

E-mail: chia-chun.lu@mpi-hd.mpg.de

\section{Michael Mayer}

Institut für Physik, Humboldt-Universität zu Berlin, Berlin, Germany.

E-mail: michael.mayerephysik.hu-berlin.de

\section{Stefan Ohm}

Deutsches Elektronen-Synchrotron (DESY), Zeuthen, Germany.

E-mail: stefan.ohm@desy.de

\section{Matthieu Renaud}

LUPM , Université Montpellier 2, CNRS/IN2P3, Montpellier, France.

E-mail: mrenaudelupm.univ-montp2.fr

\section{Jacco Vink}

Gravitation Astroparticle Physics Amsterdam (GRAPPA), Anton Pannekoek Institute for

Astronomy, University of Amsterdam, Amsterdam, Netherlands.

E-mail: j.vink@uva.nl

\section{for the H.E.S.S.Collaboration}

The Large Magellanic Cloud (LMC) is an irregular satellite galaxy of the Milky Way, which has been observed extensively at Very-High-Energy (VHE) $\gamma$ rays with the H.E.S.S. (High Energy Stereoscopic System) telescopes, obtaining a deep exposure of 210 hours. In this talk we will present the results of this campaign.

Besides the already known pulsar wind nebula N 157B, these observations establish significant VHE $\gamma$-ray emission from the super-bubble 30 Dor $\mathrm{C}$ and show evidence for emission from the supernova remnant $\mathrm{N}$ 132D. It is the first unambiguous detection of $\gamma$ rays from a super-bubble and for the first time individual cosmic-ray accelerators are identified in an external galaxy. Contrary to theoretical expectations, VHE $\gamma$-ray emission is not detected from the supernova remnant SN 1987A.

We will discuss these three objects, representing the high-energy tip of the VHE $\gamma$-ray source population in the LMC, as possible cosmic-ray accelerators, and compare them with similar systems in our Galaxy. Further discoveries can be expected with more sensitive surveys of the LMC in $\gamma$ rays, for instance with the Cherenkov Telescope Array.

These results have been published in [1].

The 34th International Cosmic Ray Conference,

30 July- 6 August, 2015

The Hague, The Netherlands 


\section{References}

[1] H.E.S.S. Collaboration, A. Abramowski, F. Aharonian, F. Ait Benkhali, A. G. Akhperjanian, E. O. Angüner, M. Backes, S. Balenderan, A. Balzer, A. Barnacka, and et al., The exceptionally powerful TeV $\gamma$-ray emitters in the Large Magellanic Cloud, Science 347 (Jan., 2015) 406-412.

${ }^{*}$ Speaker. 